\title{
Diffusion behavior of transition metals in field-assisted ion-exchanged glasses
}

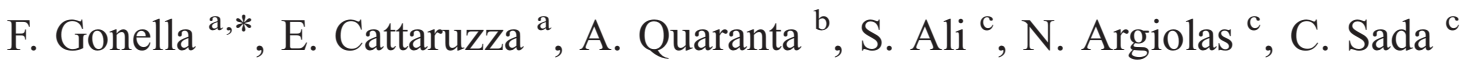 \\ ${ }^{a}$ Dip. di Chimica Fisica, Università Ca' Foscari di Venezia, Dorsoduro 2137, 30123 Venezia, Italy \\ ${ }^{\mathrm{b}}$ Dip. di Ingegneria dei Materiali, Università di Trento, via Mesiano 77, 38050 Povo (TN), Italy \\ ${ }^{\mathrm{c}}$ Dip. di Fisica, Università di Padova, via Marzolo 8, 35131 Padova, Italy
}

Received 5 May 2006; received in revised form 26 June 2006; accepted 31 July 2006

\begin{abstract}
The use of ion-exchange techniques for doping silicate glasses with transition metals has attracted much attention in the last decades for its potential in several applications, namely, light waveguides technology, luminescent materials, and for the possibility to realize systems in which metal nanocluster formation is controlled by suitable post-exchange techniques. In this framework, the control of metal distribution inside the glass is a central issue for both the understanding of the incorporation process and for the definition of effective preparation protocols. In this experiment, metallic films ( $\mathrm{Ag}, \mathrm{Cu}, \mathrm{Au}, \mathrm{Co}$ ) were deposited onto the substrates by the rf-sputtering technique. Metal ions then penetrate to substitute glass alkali by means of field-assisted ion-exchange, realized at different temperature and electric field values. In particular, we present in this paper the Au doping of silicate glasses, successfully realized for the first time with this method. The gold diffusion profiles, as measured by Secondary Ion Mass Spectrometry (SIMS), indicate that the migration depends on the experimental parameters (temperature and electric field), but also on the local structure, as well as on chemical phenomena occurring at the metal/glass interface.
\end{abstract}

(C) 2006 Elsevier B.V. All rights reserved.

Keywords: Glass-based composites; Diffusion in solids; Electromigration

\section{Introduction}

Ion-exchange process is the subject of basic and applied research for the potential exploitation of new dopants in the preparation of passive and active glasses [1]. In the ion-exchange technique, metal dopants are usually introduced into the glass by immersing the matrix in a molten salt bath containing the dopant ions [2]. They are driven into the glass due to the chemical potential gradient, and replace alkali ions of the matrix that are released into the melt. In this way, metal concentration values well beyond the solubility limits may be achieved without clustering, and proper subsequent treatments (e.g., particle or laser irradiation or heat-treatments [3]) may be effectively used to control the cluster formation. Thermal-assisted ion-exchange is on the other hand rather uneffective when dealing with mul-

\footnotetext{
* Corresponding author.

E-mail address: gonella@unive.it (F. Gonella).
}

tivalent ion species, as is the case of cobalt and gold [4]. Multivalent ions come to substitute monovalent alkali ions (mostly $\mathrm{Na}$ ) of the matrix, requiring structural modifications that come to depend crucially on the local composition of the glass.

A less explored technique uses an external electric field to assist the migration, making it possible to perform ion-exchange with multivalent ions, that are driven by the gradient in the electrochemical potential. In this configuration, the metal dopant supplier is a metallic film directly deposited on the glass matrix. No interdiffusion between the ionic species occurs in this case, where the dopant ions coming from the film replace the alkali ions of the glass matrix. At present, $\mathrm{Ag}-\mathrm{Na}$ and $\mathrm{Cu}-\mathrm{Na}$ are the most studied field-assisted processes [1,5-7], with application in the light waveguide technology and in the field of luminescent glasses. An effective phenomenological description of the dopant diffusion is still lacking for field-assisted migration of multivalent species: the diffusion coefficients and so the activation energies depend on the local ionic concentration, which is related to the local structure modification. The migration of the 
ions is expected to be governed by the dynamics of the diffusion, while the chemistry of oxoreductive equilibrium should play a negligible role, as already observed in the case of copper [8].

In this work, a field-assisted configuration (electric field up to $400 \mathrm{~V} / \mathrm{mm}$ ) was used with several metals ( $\mathrm{Cu}, \mathrm{Ag}, \mathrm{Co}$ and for the first time $\mathrm{Au}$ ), allowing the penetration from rf-sputtering deposited films to depths of the order of the micrometer, and preliminary results are presented for $\mathrm{Au}$ ion-exchange.

\section{Experimental}

Metallic films ( $\mathrm{Au}$ or $\mathrm{Co}$ ), $50 \mathrm{~nm}$ to $100 \mathrm{~nm}$ thick, were deposited onto $1 \mathrm{~mm}$ thick sodalime glass slides (wt.\% composition: $69.6 \mathrm{SiO}_{2}, 15.2 \mathrm{Na}_{2} \mathrm{O}, 1.1 \mathrm{~K}_{2} \mathrm{O}, 6.5 \mathrm{CaO}, 5.1 \mathrm{MgO}, 1.8$ $\mathrm{Al}_{2} \mathrm{O}_{3}, 0.4 \mathrm{SO}_{3}, 0.2 \mathrm{TiO}_{2}$ and 0.1 traces) in a radiofrequency magnetron sputtering deposition apparatus. Depositions on both sides of the samples were performed by means of a $13.56 \mathrm{MHz}$ radiofrequency source in a (neutral) pure Ar atmosphere, at a pressure of $50 \times 10^{-2} \mathrm{~Pa}$. The magnetron source was tilted offline to adjust the deposition focal point on the SGL substrates. After cleaning, the glass substrates were heated at $450 \mathrm{~K}$ for $30 \mathrm{~min}$ to redistribute the sodium possibly removed from the surfaces. During the deposition, the sample holder was rotated at $15 \mathrm{rpm}$ to ensure deposition homogeneity. The rf-power to the 2" diameter metal target was fixed at $50 \mathrm{~W}$, for a deposition time of $35 \mathrm{~min}$.

The samples were then ion-exchanged by means of the apparatus illustrated in Fig. 1. Different sets of samples were prepared at temperatures ranging from $200{ }^{\circ} \mathrm{C}$ to $400{ }^{\circ} \mathrm{C}$, with applied voltage values across the samples ranging from $100 \mathrm{~V}$ to $400 \mathrm{~V}$. The process time was two hours for both $\mathrm{Au}$ and $\mathrm{Co}$ ion-exchanged samples. The current density through the sample was monitored during all the exchange process. In the case of double ion-exchange experiments, a $100 \mathrm{~nm}$ thick Au film was deposited on one side of some Co-deposited samples. The rfpower to the 2 " diameter Au target was fixed at $40 \mathrm{~W}$, for a deposition time of $60 \mathrm{~min}$.

Dopants and alkali in-depth concentration profiles were determined by secondary ion mass spectrometry (SIMS) using an IMS-4f CAMECA spectrometer, equipped with a normal

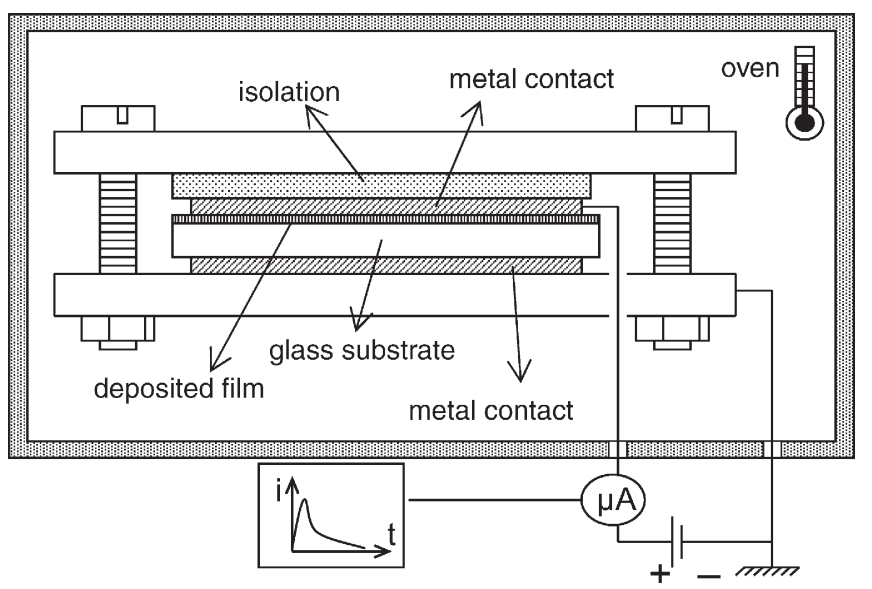

Fig. 1. Scheme of the field-assisted ion exchange apparatus. incidence electron gun to compensate the surface charge buildup while profiling insulating samples. The $4.5 \mathrm{keV}$ electrons are decelerated by an applied potential at the sample, assuring a plasma of electrons with zero velocity near the surface of the sample. When charge build-up occurs, electrons are removed from the surface so that the possible charge unbalance is exactly compensate, therefore no distortion is induced on the SIMS profiles. A $14.5 \mathrm{keV} \mathrm{Cs}^{+}$primary beam and negative secondary ion detection (rastered area: $0.125 \cdot 0.125 \mathrm{~mm}^{2}$ ) were used.

\section{Results and discussion}

The monitoring of current density across the samples during the exchange showed in most cases an increase, much more rapid in the case of gold, with a maximum of the order of few $\mathrm{mA} / \mathrm{cm}^{2}$, scarcely dependent on the applied voltage, and then decreasing monotonically until the end of the exchange time $(2 \mathrm{~h})$. The current reached its maximum after some hundred seconds from the electric field application in the case of cobalt, and much more rapidly in the case of silver and gold. In particular, in the case of gold ion-exchange, a current peak was observed in the first tens of seconds. A current increase across the samples in the first time of the process was already described in the literature $[9,10]$ for both $\mathrm{Ag}$ and $\mathrm{Cu}$ diffusion into glass from metal films. The process can be generally described in terms of an electrochemical cell, where the glass substrate acts as a solid state electrolyte, and the anode and cathode are metal films. First, a metal oxidation process occurs, so that the oxide layer at the interface can supply the ions for the exchange, thus giving rise to the in-depth diffusion. Alkali ion migration, typically much faster than incoming metal, establishes a spacecharge distribution in the glass matrix $[9,10]$, followed by oxygen diffusion towards the surface. The oxygen migration then determines the oxidation of the dopant at the metalsubstrate interface. Field-assisted diffusion of the resulting metal ions into the substrate thus depends in a complex way on both the chemical and the physical phenomena at the interface and within the glass $[11,12]$. This behavior is also related to the presence of two regimes for the current density: first, a relatively rapid migration takes place for oxygen and sodium ions of the glass, in opposite directions, and this determines the rapid increase in the current; then, the current exhibits a slow decay (without reaching zero for the used exchange times), related to the actual diffusion process of metal. The different duration of the current rise, depending on the metal, must be ascribed to the different chemical behavior of the metals at the interface. At present, no evidence for the formation of metallic or oxide nanoclusters can be supported by our experimental data: the shape of diffusion profiles indicates diffusion mechanism that do not involve the formation of clusters, a situation similar to those already reported for $\mathrm{Cu}$ and $\mathrm{Ag}$ migration $[13,14]$, for which more mobile ion species $\left(\mathrm{Cu}^{+}\right.$and $\mathrm{Ag}^{+}$) do not however give rise to clusterization, at even higher temperatures in the case of $\mathrm{Cu}$.

Besides Na, SIMS and RBS (Rutherford backscattering spectrometry) analyses evidenced migration also of the other (less mobile) alkali species of the glass matrix, namely, $\mathrm{Ca}, \mathrm{K}$ 


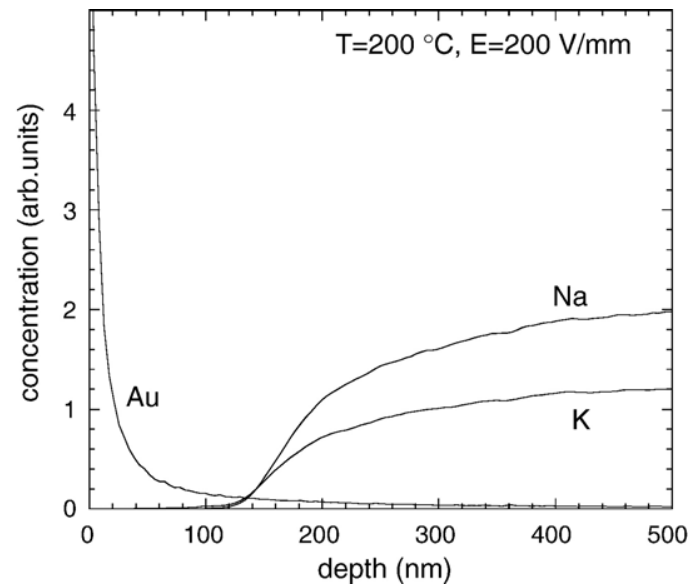

Fig. 2. SIMS concentration profiles of $\mathrm{Au}, \mathrm{Na}$ and $\mathrm{K}$ for an ion-exchanged sample treated at $T=200{ }^{\circ} \mathrm{C}$ under $E=200 \mathrm{~V} / \mathrm{mm}$ of electric field.

and Mg. As observed in Fig. 2 which shows the $\mathrm{Au}, \mathrm{Na}$ and $\mathrm{K}$ profiles after the ion-exchange, a strongly depleted region is formed between gold and alkali species that take place in the process, and this can play a significant role in the electroassisted migration mechanism, due to local charge unbalancing. No significant front mixing for gold during SIMS measurements was observed, in fact, SIMS profile (not reported) for asdeposited $\mathrm{Au} / \mathrm{glass}$ samples showed a gold penetration typically less than $10 \mathrm{~nm}$.

By increasing the applied voltage, the Au amount penetrated into the glass increases. Fig. 3 shows the Au diffusion profiles as measured for samples treated at $T=200{ }^{\circ} \mathrm{C}$ for different applied electric fields. While cobalt profiles for a corresponding experiment [15] come to resemble a Fermi function shape for high electric fields, as typical of the field-assisted process [1], $\mathrm{Au}$ diffusion profiles still exhibit a much more rapidly decreasing shape. The reason for this behavior is related to the low diffusivity of gold. Gold ions, in the +3 oxidation state, are expected to require a stronger local structural rearrangement of the glass matrix for allowing the jump in a site left free by an alkali atom.

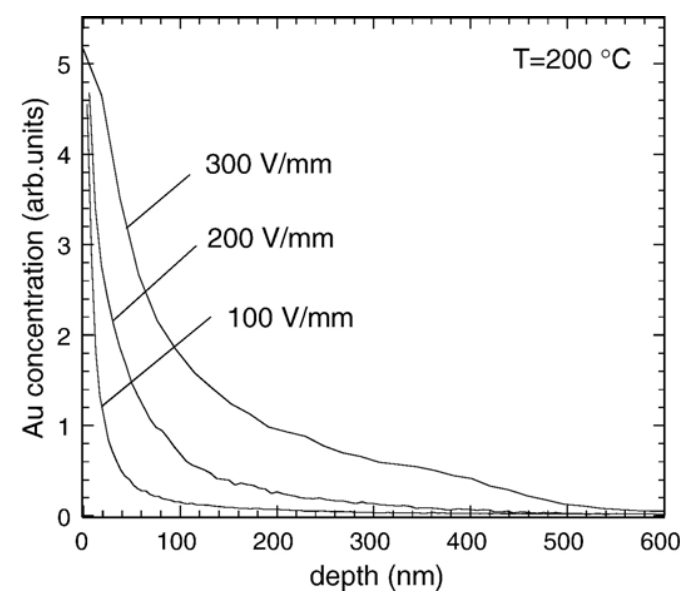

Fig. 3. SIMS concentration profiles of Au for ion-exchanged samples treated at $T=200^{\circ} \mathrm{C}$ and different applied voltage values.
An approximate description of the migration process $(x=0$ is the sample surface) can be made using the equation for the gold ions flux $J(x, t)$ :

$J(x, t)=-D \frac{\partial c}{\partial x}+\operatorname{SI}(t) c(x, t)$

where $c(x, t)$ is the local gold concentration, $D$ is the gold diffusion coefficient, $S$ is a coefficient proportional to the driving electric field and $I(t)$ is the current recorded during the treatment. The shape of the diffusion profiles evidenced that even with such simple expression a diffusion coefficient dependent on the concentration is needed, with an exponential decrease $D(x)=D_{0} \exp (-c(x))$, with the dependence related to a concentration-dependent activation energy [8]. The decrease is actually due to stronger rearrangement at high gold concentrations, leading to the formation of a network where the gold diffusivity is lower. Fig. 4 shows the fit of the experimental profile obtained for an external field $E=300 \mathrm{~V} / \mathrm{mm}$ at $T=200{ }^{\circ} \mathrm{C}$. The fit was calculated by a standard recursive finite difference integration of the standard equation for the diffusion:

$\frac{\partial c}{\partial t}=-\frac{\partial J}{\partial x}$

The parameters for the fits were $D_{0}=8 \times 10^{-14} \mathrm{~cm}^{2} / \mathrm{s}, S=$ $3 \times 10^{-6} \mathrm{~cm} / \mathrm{sA}$. It is worth remarking that even for this case the diffusion profile is not well reproduced by a classical erfc-like solution.

As observed for the Co case [15], the amount of ions penetrated significantly increases with the applied voltage, with a corresponding shift of the penetration depth, which is anyway limited by the very low diffusion coefficient. Moreover, the strong migration of both $\mathrm{Na}$ and $\mathrm{K}$ beyond the Au penetration depth (see Fig. 2) can contribute to further lowering the diffusion parameters of the incoming ions into the depleted region.

By increasing the process temperature from $200{ }^{\circ} \mathrm{C}$ to $300{ }^{\circ} \mathrm{C}$ at the fixed applied voltage of $300 \mathrm{~V}$, an accumulation of gold is observed under the surface, located in the first hundreds of nanometers. Fig. 5 shows this behavior comparing the $\mathrm{Au}$ profiles for two samples treated under a field $E=300 \mathrm{~V} / \mathrm{mm}$ for

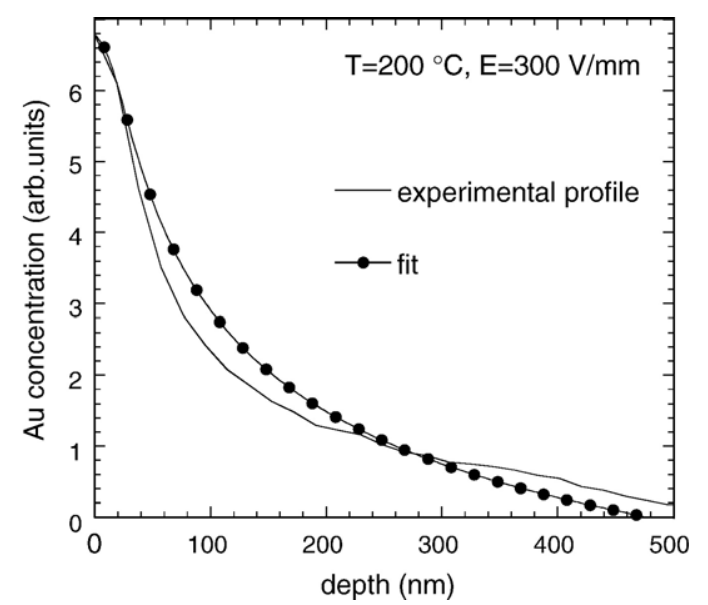

Fig. 4. Au concentration profile (solid line) and fit (dots) for a sample ionexchanged at $T=200^{\circ} \mathrm{C}$ under $E=300 \mathrm{~V} / \mathrm{mm}$ of electric field. 
different temperature values. By increasing the temperature, the occupation by the gold ions at the metal/glass interface of the sites left by alkali species is expected to be strongly enhanced, while the subsequent diffusion into the matrix is still too slow to maintain a diffusive regime typical of lower temperature (see Fig. 3). An accumulation then occurs, which further alters the diffusion coefficient - which is expected to be dependent on the local gold concentration - and the charge balancing, giving rise also to the build up of local fields effects. During the ionexchange process the glass network undergoes concentrationdependent structural changes, due to the local coordination rearrangements at the ion sites $[12,16,17]$. The diffusion coefficients therefore come to depend on the local concentration [18], giving rise to correlation effects between ionic fluxes similar to a mixed-alkali effect, where the transport parameters of the ion species may dramatically depend on the concentration $[19,20]$. The Au electro-assisted migration presented in this work cannot be described by the phenomenological approaches developed for describing other ion-exchange processes [8,21]. In fact, models usually assume the local charge neutrality, based on the constancy of the overall ionic concentration throughout the modified layer. This hypothesis cannot be supported in the case of Au migration, due to the presence of a strongly depleted region, where the charge neutrality is supposed to be due to some kind of structural network modification, similar to that observed in poled glasses.

In the case of electromigration from a solid state film, compositional analyses evidenced the formation of this depleted region, where the local charge can be balanced only by some charged defects produced under the electric field or at the filmglass interface. For all these reasons, the electromigration should be correctly described by a set of equations accounting for the change of the local microstructure and the flux of charged defects. An exhaustive picture is still lacking, and a more detailed investigation on the state of gold (depending on the experimental parameters as well as on the penetration depth) is in progress, by means of X-ray absorption fine structure (EXAFS) experiments.

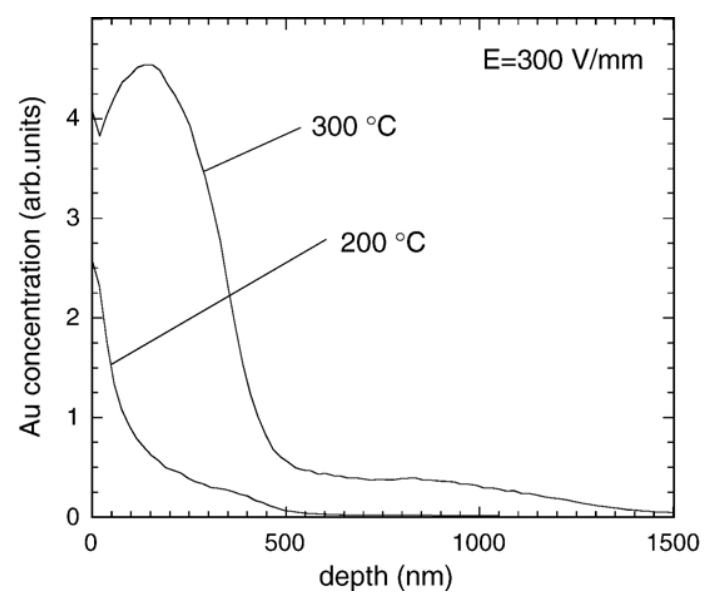

Fig. 5. SIMS concentration profiles of Au for ion-exchanged samples treated at different temperatures under $E=300 \mathrm{~V} / \mathrm{mm}$ of electric field.

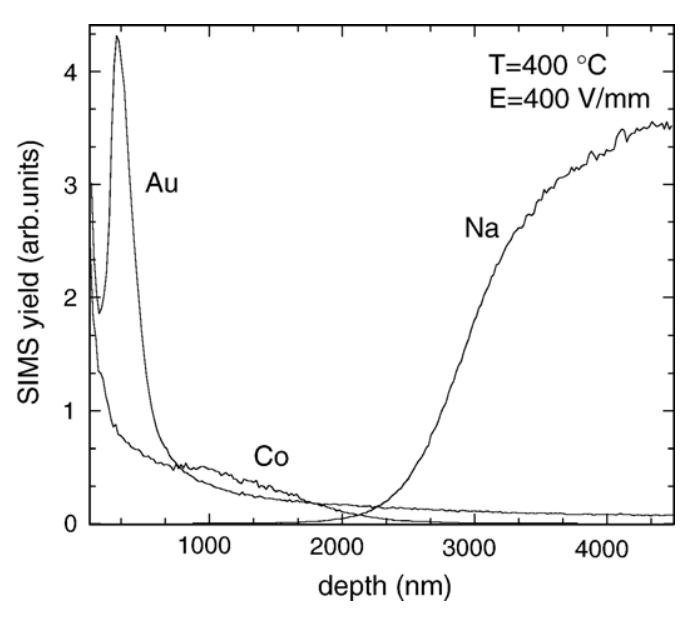

Fig. 6. SIMS concentration profiles of $\mathrm{Co}, \mathrm{Au}$ and $\mathrm{Na}$ for a double-ionexchanged sample treated at $T=400{ }^{\circ} \mathrm{C}$ under $E=400 \mathrm{~V} / \mathrm{mm}$ of electric field.

It is worth remarking that both alkali accumulation and reduction are expected to occur at the cathode interface [10], but this should not affect the depletion region near the goldexchanged region, since poling local effects are in this region much more relevant.

Preliminary experiments were also performed to explore the possibility to co-dope the glass with two metal species, with the aim to promote, by means of subsequent treatments, the formation of either core-shell or alloy nanoclusters [3]. Fig. 6 shows the first example of $\mathrm{Au} / \mathrm{Co}$ doped silicate glass obtained by field-assisted migration of the two metals. The experiment, for which a $100 \mathrm{~nm}$ thick gold layer was deposited onto the Co layer previously deposited onto the glass, was realized at $T=400{ }^{\circ} \mathrm{C}$ and at $400 \mathrm{~V}$ of applied voltage. It is clear that both metals penetrate into the matrix, following diffusion regimes that depend in a quite complex way on the external parameters and on the local structure and composition within the glass. In particular, $\mathrm{Au}$ profile exhibits a peak under the surface, suggesting some kind of diffusion-limiting phenomenon, to be further investigated. Moreover, a possible interaction of the two metals (interdiffusion, alloy formation) cannot be ruled out, as already observed in a similar configuration for $\mathrm{Cu}$ and $\mathrm{Au}$ [7], so further complicating the possibility of controlling the process. Work is in progress to select suitable configurations for the two metal films and for the experimental parameters, in order to create the condition for the controlled clusterization of complex nanostructures.

\section{Conclusions}

Gold-containing silicate glasses were obtained for the first time by a field-assisted ion-exchange method. This has been demonstrated to be a suitable technique for the Au doping of thick glass layers.

$\square$ The amount of Au that penetrated into the glass matrix, as well as the shape of the diffusion profiles, depend strongly on the process parameters, namely, applied voltage and temperature.

$\square$ Chemical phenomena occurring at the metal/glass interface play a significant role in the penetration of the Au ions coming 
from the film, while the diffusion into the glass depends on the local concentration of the ion species involved in the process.

The usual phenomenological approaches describing the ion diffusion in glass cannot be directly applied to the case $\mathrm{Au}$ ion-exchange. In particular, the role of the alkali depletion zone is to be investigated in details.

Work is in progress to provide a structural investigation of the samples, and then to use thermal or ion-beam-based treatments for promoting the clusterization.

\section{Acknowledgement}

This work has been partially supported by the Italian Ministry of Scientific Research (PRIN 2004).

\section{References}

[1] S.I. Najafi, Introduction to Glass Integrated Optics, The Artech House Optoelectronics Library, Boston, 1992.

[2] R.V. Ramaswamy, R. Srivastava, IEEE J. Lightwave Technol. 6 (1988) 984.

[3] F. Gonella, P. Mazzoldi, in: H.S. Nalwa (Ed.), Handbook of Nanostructured Materials and Nanotechnology, vol. 4, Academic Press, S. Diego, 2000 , p. 81.

[4] V.A. Zhabrev, S.I. Sviridov, Glass Phys. Chem. 29 (2003) 140.

[5] N. Valles-Villareal, A. Villalobos, H. Márquez, IEEE J. Lightwave Technol. 17 (1999) 606
[6] H. Márquez, D. Salazar, A. Villalobos, G. Paez, J.Ma. Rincon, Appl. Opt. 34 (1995) 5817.

[7] R. Oven, M. Yin, P.A. Davies, J. Phys. D: Appl. Phys. 37 (2004) 2207.

[8] F. Gonella, A. Quaranta, S. Padovani, C. Sada, F. D'Acapito, C. Maurizio, G. Battaglin, E. Cattaruzza, Appl. Phys., A 81 (2005) 1065.

[9] D. Kapila, J.L. Plawsky, AIChE J. 39 (1993) 1186.

[10] D. Kapila, J.L. Plawsky, Chem. Eng. Sci. 50 (1995) 2589.

[11] A. Quaranta, F. Gonella, J. Non-Cryst. Solids 192 and 193 (1995) 334.

[12] C. Thévenin-Annequin, M. Levy, T. Pagnier, Solid State Ionics 80 (1995) 175.

[13] F. Gonella, L.D. Bogomolova, F. D’Acapito, A. Quaranta, J. Appl. Phys. 83 (1998) 1200.

[14] E. Borsella, G. Battaglin, M.A. Garcìa, F. Gonella, P. Mazzoldi, R. Polloni, A. Quaranta, Appl. Phys., A 71 (2000) 125.

[15] F. Gonella, P. Canton, E. Cattaruzza, A. Quaranta, C. Sada, A. Vomiero, Mater. Sci. Eng. C 26 (2006) 1087.

[16] G.N. Greaves, S.J. Gurman, C.R. Catlows, A.V. Chadwick, S.N. HoudeWalter, C.M.B. Henderson, B.R. Dobson, Philos. Mag., B 64 (1991) 1059.

[17] F. D'Acapito, S. Colonna, S. Mobilio, G. Gonella, E. Cattaruzza, P. Mazzoldi, Appl. Phys. Lett. 71 (1997) 261

[18] J.M. Inman, S.N. Houde-Walter, B.L. McIntyre, Z.M. Liao, R.S. Parker, V. Simmons, J. Non-Cryst. Solids 194 (1996) 85.

[19] G.N. Greaves, K.L. Nagai, Phys. Rev., B 52 (1995) 6358

[20] G. De Marchi, G. Mazzoldi, A. Miotello, J. Non-Cryst. Solids 123 (1990) 321.

[21] F. Gonella, A. Quaranta, E. Cattaruzza, S. Padovani, C. Sada, F. D’Acapito, C. Maurizio, Computat. Mater. Sci. 33 (2005) 31. 